\title{
Synthesis and Characterization of Novel Cinnamamide Deriverties and Their Antimicrobial Activities
}

\author{
S. B. Borul ${ }^{1}$ and S. V. Agarkar ${ }^{2}$ \\ ${ }^{I}$ (Department of Chemistry, Late Ku. D.K. Banmeru Science College, Lonar Maharashtra [India]) \\ ${ }^{2}$ (Anuradha Engineering College, Chikhli Maharashtra [India])
}

\begin{abstract}
Cinnamamides and its derivatives possess wide range of biological activities, and thus novel cinnamamide derivatives were synthesized by using heterocyclic moiety. All the compounds were characterized by on the basis of analytical data, IR, NMR spectral studies. Biological screening of the synthesized compounds is most important task which decides the practical utility of compounds in various fields such as medicinal and pharmaceuticals.The synthesized compounds antimicrobial activities were also evaluated. The preliminary results showed that all the title compounds had certain antimicrobial activities against S. aureus, Bacillus, and E.coli. at a different concentration.
\end{abstract}

Key Words: Aldehydes, Antimicrobial activities, Cinnamamides, Heterocyclic moiety, synthesis.

\section{Introduction}

Cinnamamides and its derivatives possess wide range of activities in several fields. Such as medicinal, pharmaceuticals, agricultural, biological ${ }^{[1]}$ and many other activities. In medicinal field several Cinnamamides and their derivatives are reported to show central nervous system depressant, anticonvulsant ${ }^{[2]}$, muscle relaxant, antiallergic, antineoplastic, antitumor, anesthetic, analgesic and anti-infective activities ${ }^{[3-7]}$, etc. In the agrochemical field, insecticidal, their avian repellent, herbicidal activities, and several excellent cinnamamide fungicides ${ }^{[8-9]}$ for example dimethomorph, fluormorph and pyrimorph, have been successfully developed.

Biological screening of the synthesized compounds is most important task which decides the practical utility of compounds in various fields such as medicinal, pharmaceuticals etc. Inspire of wide range of their applications and very less attention is paid towards the synthesis of cinnamamides derivatives containing heterocyclic moiety. Literature survey and biological activities' of cinnamamides have motivated to undertake the synthesized novel cinnamamides entitled "(2E)-1-(4-methylpeperazin-1-yl)-3-subsituted phenylprop-2-en-1one Cinnamamides. The present study is related to the synthesis of some novel cinnamamides containing heterocyclic moiety. Synthesized compounds were characterized by elemental analysis and IR, NMR spectral studies. The preliminary results showed that all the title compounds had certain antimicrobial activities against S. aureus, Bacillus, and E.coli. at a different concentration.

\section{Result And Discussion}

All synthesized novel cinnamamides compounds contained heterocyclic moiety in the form of $\mathrm{N}$ methyl Piperazine. The Witting reaction is an important method for the synthesis of alkenes. By using this method novel cinnamamides containing heterocyclic moiety entitled (2E)-1-(4-methylpeperazin-1-yl)-3substituted phenylprop-2-en-1-one Cinnamamides are synthesized from different aromatic aldehydes and Witting reagents having good yields. The yields of synthesized compounds were ranging from 54 to $78 \%$. All synthesized compounds were characterized on the basis of melting point, elemental analysis, Rf value, IR spectra and 1HNMR spectral analysis. Preliminary results showed that all the title compounds had certain antimicrobial activities against S. aureus, Bacillus, and E.coli. at a different concentration.

\section{Experimental}

3.1 Synthesis of Wittig reagent - N-methyl piperazine chloracetamide were synthesized by using equimolar solution of chloroacetylchloride and $\mathrm{N}$-methyl piperazine in chloroform at $0^{\circ} \mathrm{C}$ with continuous stirring in fuming chamber. When this reaction mixture gives the salt by adding its solution in benzene to the stirred solution of triphenylphosphine and reaction mixture was refluxed for 4-6 hrs. The solid products obtained were filtered and air dried. Thus for Purification obtained salt was dissolved in $100 \mathrm{ml}$ water then $90 \mathrm{ml}$ of dry benzene, add 1-2 drops of phenolphthalein indicator and add $\mathrm{NaOH}$ solution in it till pink colour persist this was indicates that the neutralization of present acid from reagent. Then benzene layer was separated and washed with water and concentrated to one third volume. Finally the product scratched with n-Hexane to obtain solid witting reagent. 


\subsection{Synthesis of (2E)-1-(4-methylpeperazin-1-yl)-3-Substituted phenylprop-2-en-1-one Cinnamamides -}

Equimolar solution of Witting reagent and various aromatic aldehydes in water and refluxed for 4 to 6 hrs. The progress of reaction was monitored by thin layer chromatography. Melting points were taken by open capillary method. The elemental analysis was calculated for carbon, hydrogen, nitrogen and chlorine. IR spectra were recorded with TMS as internal standard using $\mathrm{CDCl} 3$. All Synthesized compounds were purified by column chromatography. All chemicals used were of analytical grade.

\section{Antimicrobial Activities}

Antimicrobial activities of newly synthesized compounds were carried out by using cup plate agar diffusion method at $01 \mathrm{mg} / \mathrm{ml}, 0.5 \mathrm{mg} / \mathrm{ml}$, and $0.25 \mathrm{mg} / \mathrm{ml}$ in DMSO against using antibiotics ciprofloxacin. Plates were incubated $24 \mathrm{hrs}$ at $37^{\circ} \mathrm{C}$ and zone of inhibition were measured in mm. Result have been incorporated in table, all synthesized compound were found to be moderately active against bacteria.

5.1 Synthesis of witting reagent-

\section{Schemes}<smiles>COC(=O)CCl</smiles>

2-chloro-1-(4-methylpiperazin-1-yl) ethanone

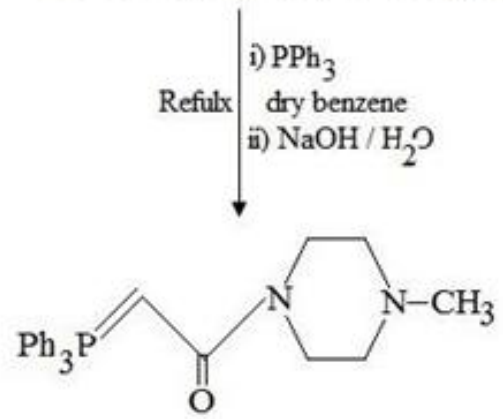

Wittig reagent

5.2 Synthesis of Cinnamamides derivatives of heterocyclic moiety-<smiles></smiles><smiles>[R]c1c([R])c([R])c(/C=C/C(=O)N2CCN(C)CC2)c([R])c1[Y]</smiles>

Substituted Aldehyde

(2E)-1-(4-methylpiperazin-1-yl)-3-subtituted phenylprop-2-en-1-one cinnamamides

VI. Tables

Table No. 1 Antimicrobial activities of the compounds (in mm)

\begin{tabular}{|c|c|c|c|c|c|c|c|c|c|}
\hline \multirow{2}{*}{ Compounds } & \multicolumn{3}{|c|}{ S. aureus } & \multicolumn{3}{c|}{ Bacillus } & \multicolumn{3}{c|}{ E.coli } \\
\cline { 2 - 10 } & $1 \mathrm{Mg} / \mathrm{ml}$ & $0.5 \mathrm{Mg} / \mathrm{ml}$ & $0.25 \mathrm{Mg} / \mathrm{ml}$ & $1 \mathrm{Mg} / \mathrm{ml}$ & $0.5 \mathrm{Mg} / \mathrm{ml}$ & $0.25 \mathrm{Mg} / \mathrm{ml}$ & $1 \mathrm{Mg} / \mathrm{ml}$ & $0.5 \mathrm{Mg} / \mathrm{ml}$ & $0.25 \mathrm{Mg} / \mathrm{ml}$ \\
\hline Ia & 14 & 13 & 11 & 12 & 10 & 09 & 13 & 11 & 10 \\
\hline Ib & 15 & 14 & 12 & 14 & 13 & 12 & 18 & 17 & 15 \\
\hline Ic & 18 & 17 & 14 & 13 & 12 & 10 & 15 & 13 & 12 \\
\hline Id & 15 & 14 & 12 & 21 & 18 & 16 & 19 & 17 & 16 \\
\hline Ie & 12 & 10 & 09 & 18 & 17 & 16 & 12 & 11 & 10 \\
\hline If & 17 & 16 & 15 & 31 & 28 & 25 & 16 & 15 & 12 \\
\hline Ig & 23 & 19 & 12 & 15 & 12 & 13 & 16 & 14 & 10 \\
\hline Ih & 24 & 22 & 21 & 18 & 16 & 17 & 18 & 15 & 16 \\
\hline Ii & 20 & 18 & 14 & 24 & 21 & 19 & 30 & 28 & 24 \\
\hline Ij & 24 & 22 & 21 & 19 & 15 & 12 & 29 & 26 & 22 \\
\hline
\end{tabular}


Table No.2-Substituted aromatic aldehydes used in the synthesis of Cinnamamides

\begin{tabular}{|c|c|c|c|c|c|c|}
\hline $\begin{array}{l}\text { Sr. } \\
\text { No. }\end{array}$ & Substituent & $\mathrm{R} 1$ & $\mathrm{R} 2$ & $\mathrm{R} 3$ & $\mathrm{R} 4$ & R5 \\
\hline 1 & Ia & $\mathrm{H}$ & $\mathrm{H}$ & $\mathrm{H}$ & $\mathrm{H}$ & $\mathrm{H}$ \\
\hline 2 & $\mathrm{Ib}$ & $\mathrm{H}$ & $\mathrm{H}$ & $\mathrm{OMe}$ & $\mathrm{H}$ & $\mathrm{H}$ \\
\hline 3 & Ic & $\mathrm{H}$ & $\mathrm{OMe}$ & $\mathrm{OMe}$ & $\mathrm{H}$ & $\mathrm{H}$ \\
\hline 4 & Id & $\mathrm{H}$ & $\mathrm{OMe}$ & $\mathrm{OMe}$ & $\mathrm{OMe}$ & $\mathrm{H}$ \\
\hline 5 & Ie & $\mathrm{H}$ & \multicolumn{2}{|c|}{$-\mathrm{O}-\mathrm{CH}_{2}-\mathrm{O}-$} & $\mathrm{H}$ & $\mathrm{H}$ \\
\hline 6 & If & $\mathrm{NO}_{2}$ & $\mathrm{H}$ & $\mathrm{H}$ & $\mathrm{H}$ & $\mathrm{H}$ \\
\hline 7 & $\mathrm{Ig}$ & $\mathrm{H}$ & $\mathrm{H}$ & $\mathrm{Cl}$ & $\mathrm{H}$ & $\mathrm{H}$ \\
\hline 8 & Ih & $\mathrm{H}$ & $\mathrm{H}$ & $\mathrm{NO}_{2}$ & $\mathrm{H}$ & $\mathrm{H}$ \\
\hline 9 & Ii & $\mathrm{H}$ & $\mathrm{H}$ & $\mathrm{N}(\mathrm{Me})_{2}$ & $\mathrm{H}$ & $\mathrm{H}$ \\
\hline 10 & $\mathrm{Ij}$ & $\mathrm{H}$ & $\mathrm{H}$ & $\mathrm{OH}$ & $\mathrm{H}$ & $\mathrm{H}$ \\
\hline
\end{tabular}

Table No.3- Characteristics data for synthesized Cinnamamides

\begin{tabular}{|c|c|c|c|c|c|}
\hline Sr. No. & Compounds & Molecular Formula & Molecular Wt. & Yield \% & M.P. ${ }^{0} \mathrm{C}$ \\
\hline 1 & $\mathrm{Ia}$ & $\mathrm{C}_{14} \mathrm{H}_{18} \mathrm{ON}_{2}$ & 230 & 76 & 69 \\
\hline 2 & $\mathrm{Ib}$ & $\mathrm{C}_{15} \mathrm{H}_{20} \mathrm{O}_{2} \mathrm{~N}_{2}$ & 260 & 62 & 165 \\
\hline 3 & $\mathrm{Ic}$ & $\mathrm{C}_{16} \mathrm{H}_{22} \mathrm{O}_{3} \mathrm{~N}_{2}$ & 290 & 54 & 205 \\
\hline 4 & $\mathrm{Id}$ & $\mathrm{C}_{17} \mathrm{H}_{24} \mathrm{O}_{4} \mathrm{~N}_{2}$ & 320 & 66 & 172 \\
\hline 5 & $\mathrm{I}$ & $\mathrm{C}_{15} \mathrm{H}_{18} \mathrm{O}_{3} \mathrm{~N}_{3}$ & 288 & 58 & 102 \\
\hline 6 & If & $\mathrm{C}_{14} \mathrm{H}_{17} \mathrm{O}_{3} \mathrm{~N}_{2}$ & 261 & 78 & 79 \\
\hline 7 & $\mathrm{Ig}$ & $\mathrm{C}_{14} \mathrm{H}_{17} \mathrm{ON}_{2} \mathrm{Cl}$ & 264.5 & 70 & 218 \\
\hline 8 & $\mathrm{Ih}$ & $\mathrm{C}_{14} \mathrm{H}_{17} \mathrm{O}_{3} \mathrm{~N}_{3}$ & 275 & 56 & 200 \\
\hline 9 & $\mathrm{Ii}$ & $\mathrm{C}_{16} \mathrm{H}_{23} \mathrm{ON}_{2}$ & 259 & 64 & 82 \\
\hline 10 & $\mathrm{Ij}$ & $\mathrm{C}_{14} \mathrm{H}_{18} \mathrm{O}_{2} \mathrm{~N}_{2}$ & 246 & & 52 \\
\hline
\end{tabular}

Table No.4-Elemental analysis of synthesized compounds

\begin{tabular}{|c|c|c|c|c|c|c|}
\hline Compounds & $\begin{array}{l}\text { Molecular } \\
\text { Formula }\end{array}$ & $\% \mathrm{C}$ & $\% \mathrm{H}$ & $\% \mathrm{O}$ & $\% \mathrm{~N}$ & $\% \mathrm{Cl}$ \\
\hline Ia & $\mathrm{C}_{14} \mathrm{H}_{18} \mathrm{ON}_{2}$ & $\begin{array}{c}73.06 \\
(73.04) \\
\end{array}$ & $\begin{array}{c}7.88 \\
(7.83) \\
\end{array}$ & $\begin{array}{c}7.00 \\
(6.96) \\
\end{array}$ & $\begin{array}{c}12.19 \\
(12.17) \\
\end{array}$ & --- \\
\hline $\mathrm{Ib}$ & $\mathrm{C}_{15} \mathrm{H}_{20} \mathrm{O}_{2} \mathrm{~N}_{2}$ & $\begin{array}{c}69.26 \\
(69.23)\end{array}$ & $\begin{array}{c}7.72 \\
(7.69)\end{array}$ & $\begin{array}{c}12.36 \\
(12.31)\end{array}$ & $\begin{array}{c}10.82 \\
(10.77)\end{array}$ & --- \\
\hline Ic & $\mathrm{C}_{16} \mathrm{H}_{22} \mathrm{O}_{3} \mathrm{~N}_{2}$ & $\begin{array}{c}66.26 \\
(66.21)\end{array}$ & $\begin{array}{c}7.64 \\
(7.59)\end{array}$ & $\begin{array}{c}16.62 \\
(16.55)\end{array}$ & $\begin{array}{c}9.70 \\
(9.66)\end{array}$ & --- \\
\hline Id & $\mathrm{C}_{17} \mathrm{H}_{24} \mathrm{O}_{4} \mathrm{~N}_{2}$ & $\begin{array}{c}63.80 \\
(63.75)\end{array}$ & $\begin{array}{c}7.54 \\
(7.50)\end{array}$ & $\begin{array}{c}20.06 \\
(20.00)\end{array}$ & $\begin{array}{c}8.80 \\
(8.75)\end{array}$ & --- \\
\hline $\mathrm{Ie}$ & $\mathrm{C}_{15} \mathrm{H}_{18} \mathrm{O}_{3} \mathrm{~N}_{3}$ & $\begin{array}{c}62.58 \\
(62.50)\end{array}$ & $\begin{array}{c}6.30 \\
(6.25) \\
\end{array}$ & $\begin{array}{c}16.71 \\
(16.66)\end{array}$ & $\begin{array}{c}14.62 \\
(14.58)\end{array}$ & --- \\
\hline If & $\mathrm{C}_{14} \mathrm{H}_{17} \mathrm{O}_{3} \mathrm{~N}_{2}$ & $\begin{array}{c}64.42 \\
(64.36)\end{array}$ & $\begin{array}{c}6.53 \\
(6.51)\end{array}$ & $\begin{array}{c}18.42 \\
(18.39)\end{array}$ & $\begin{array}{c}10.75 \\
(10.72)\end{array}$ & --- \\
\hline Ig & $\mathrm{C}_{14} \mathrm{H}_{17} \mathrm{ON}_{2} \mathrm{Cl}$ & $\begin{array}{c}64.02 \\
(63.51)\end{array}$ & $\begin{array}{c}6.48 \\
(6.42)\end{array}$ & $\begin{array}{c}6.10 \\
(6.05)\end{array}$ & $\begin{array}{c}10.56 \\
(10.58)\end{array}$ & $\begin{array}{c}13.40 \\
(13.42)\end{array}$ \\
\hline Ih & $\mathrm{C}_{14} \mathrm{H}_{17} \mathrm{O}_{3} \mathrm{~N}_{3}$ & $\begin{array}{c}62.12 \\
(61.10) \\
\end{array}$ & $\begin{array}{c}6.21 \\
(6.18) \\
\end{array}$ & $\begin{array}{c}17.46 \\
(17.45) \\
\end{array}$ & $\begin{array}{c}15.28 \\
(15.27)\end{array}$ & --- \\
\hline Ii & $\mathrm{C}_{16} \mathrm{H}_{23} \mathrm{ON}_{2}$ & $\begin{array}{c}74.16 \\
(74.13)\end{array}$ & $\begin{array}{c}8.90 \\
(8.88)\end{array}$ & $\begin{array}{c}6.20 \\
(6.17) \\
\end{array}$ & $\begin{array}{c}10.84 \\
(10.81) \\
\end{array}$ & --- \\
\hline $\mathrm{Ij}$ & $\mathrm{C}_{14} \mathrm{H}_{18} \mathrm{O}_{2} \mathrm{~N}_{2}$ & $\begin{array}{c}68.31 \\
(68.29)\end{array}$ & $\begin{array}{c}7.32 \\
(7.31)\end{array}$ & $\begin{array}{c}13.03 \\
(13.00)\end{array}$ & $\begin{array}{c}11.40 \\
(11.38)\end{array}$ & --- \\
\hline
\end{tabular}

In bracket calculated percentages of element.

\section{Spectral Data Studies}

$\mathbf{I a}=(2 \mathrm{E})-1$-(4-methylpeperazin-1-yl)-3- phenylprop-2-en-1-one Cinnamamides-

IR $\left(\mathrm{cm}^{-1}\right) 1656,1595$

1H NMR-( $\delta)-2.2(\mathrm{~s}),(3 \mathrm{H}), \mathrm{NMe} ; 2.9(\mathrm{t}),(4 \mathrm{H}) ; 3.5(\mathrm{t}),(4 \mathrm{H}) ; 6.7(\mathrm{~d}),(1 \mathrm{H}),(\mathrm{CH}=\mathrm{CHCO}), \mathrm{J}=15.94 \mathrm{HZ} ; 6.8(\mathrm{~d}),(1 \mathrm{H})$, $\left(\mathrm{CH}=\mathrm{CHC}_{6} \mathrm{H}_{5}\right) \mathrm{J}=15.94 \mathrm{HZ} ; 7.2-7.5(\mathrm{~m}),(5 \mathrm{H}),\left(\mathrm{C}_{6} \mathrm{H}_{5}\right)$.

Ib- IR $\left(\mathrm{cm}^{-1}\right) 1685,1600$

1H NMR- $(\delta)-2.2(\mathrm{~s}),(3 \mathrm{H}), \mathrm{NMe} ; 2.9(\mathrm{t}),(4 \mathrm{H}) ; 3.5(\mathrm{t}),(4 \mathrm{H}) ; 6.9(\mathrm{~d}),(1 \mathrm{H}),(\mathrm{CH}=\mathrm{CHCO}), \mathrm{J}=15.60 \mathrm{HZ} ; 7.0(\mathrm{~d}),(1 \mathrm{H})$, $\left(\mathrm{CH}=\mathrm{CHC}_{6} \mathrm{H}_{5}\right) \mathrm{J}=15.60 \mathrm{HZ} ; 3.2(\mathrm{~s}),(3 \mathrm{H}),(\mathrm{OMe}) ; 7.2(\mathrm{~d}),(2 \mathrm{H}) ; 7.4(\mathrm{~d}),(2 \mathrm{H})$.

Ic- IR $\left(\mathrm{cm}^{-1}\right) 1678,1644$

1H NMR- $(\delta)-2.2(\mathrm{~s}),(3 \mathrm{H}), \mathrm{NMe} ; 2.9(\mathrm{t}),(4 \mathrm{H}) ; 3.5(\mathrm{t}),(4 \mathrm{H}) ; 6.9(\mathrm{~d}),(1 \mathrm{H}),(\mathrm{CH}=\mathrm{CHCO}), \mathrm{J}=15.70 \mathrm{HZ} ; 7.2(\mathrm{~d}),(1 \mathrm{H})$, $\left(\mathrm{CH}=\mathrm{CHC}_{6} \mathrm{H}_{5}\right) \mathrm{J}=15.70 \mathrm{HZ} ; 3.2(\mathrm{~s}),(6 \mathrm{H}),(\mathrm{OMe}) ; 6.9-7.1(\mathrm{~m}),(3 \mathrm{H}), \mathrm{Ar}-\mathrm{H}$.

Id- IR $\left(\mathrm{cm}^{-1}\right) 1688,1636$

1H NMR-( $\delta)-2.2(\mathrm{~s}),(3 \mathrm{H}), \mathrm{NMe} ; 2.9(\mathrm{t}),(4 \mathrm{H}) ; 3.5(\mathrm{t}),(4 \mathrm{H}) ; 6.9(\mathrm{~d}),(1 \mathrm{H}),(\mathrm{CH}=\mathrm{CHCO}), \mathrm{J}=15.8 \mathrm{HZ} ; 7.4(\mathrm{~d}),(1 \mathrm{H})$, $\left(\mathrm{CH}=\mathrm{CHC}_{6} \mathrm{H}_{5}\right) \mathrm{J}=15.84 \mathrm{HZ}$; 3.2(s), $(9 \mathrm{H}),(\mathrm{OMe}) ; 6.6(\mathrm{~s}),(2 \mathrm{H}), \mathrm{Ar}-\mathrm{H}$. 
Ie-IR $\left(\mathrm{cm}^{-1}\right) 1670,1650$

1HNMR-1H NMR-(ס)-2.2(s), (3H), NMe; 2.9(t), $(4 \mathrm{H}) ; 3.5(\mathrm{t}),(4 \mathrm{H}) ; 6.9(\mathrm{~d}),(1 \mathrm{H}),(\mathrm{CH}=\mathrm{CHCO}), \mathrm{J}=15.84 \mathrm{HZ}$; 7.4(d), $(1 \mathrm{H}),\left(\mathrm{CH}=\mathrm{CHC}_{6} \mathrm{H}_{5}\right) \mathrm{J}=15.84 \mathrm{HZ} ; 4.8(\mathrm{~s}),(4 \mathrm{H}),\left(\mathrm{H}_{2} \mathrm{COCH}_{2}\right)$; 7.4-7.6(s), $(3 \mathrm{H}), \mathrm{Ar}-\mathrm{H}$.

If- IR $\left(\mathrm{cm}^{-1}\right) 1664,1680$

1H NMR- $(\delta)-2.2(\mathrm{~s}),(3 \mathrm{H}), \mathrm{NMe} ; 2.9(\mathrm{t}),(4 \mathrm{H}) ; 3.5(\mathrm{t}),(4 \mathrm{H}) ; 6.8(\mathrm{~d}),(1 \mathrm{H}),(\mathrm{CH}=\mathrm{CHCO}), \mathrm{J}=15.72 \mathrm{HZ} ; 7.3(\mathrm{~d}),(1 \mathrm{H})$, $\left(\mathrm{CH}=\mathrm{CHC}_{6} \mathrm{H}_{5}\right) \mathrm{J}=15.72 \mathrm{HZ} ; 7.4-7.5(\mathrm{~m}),(4 \mathrm{H}), \mathrm{Ar}-\mathrm{H} .1 \mathrm{H}$

Ig- IR $\left(\mathrm{cm}^{-1}\right) 1656,1580$

1H NMR- $(\delta)-2.2(\mathrm{~s}),(3 \mathrm{H}), \mathrm{NMe} ; 2.9(\mathrm{t}),(4 \mathrm{H}) ; 3.5(\mathrm{t}),(4 \mathrm{H}) ; 6.6(\mathrm{~d}),(1 \mathrm{H}),(\mathrm{CH}=\mathrm{CHCO}), \mathrm{J}=15.70 \mathrm{HZ} ; 6.8(\mathrm{~d}),(1 \mathrm{H})$, $\left(\mathrm{CH}=\mathrm{CHC}_{6} \mathrm{H}_{5}\right) \mathrm{J}=15.70 \mathrm{HZ} ; 7.3-7.5(\mathrm{~m}),(4 \mathrm{H})$.

Ih- IR $\left(\mathrm{cm}^{-1}\right) 1664,1680$

1H NMR-( $\delta)-2.2(\mathrm{~s}),(3 \mathrm{H}), \mathrm{NMe} ; 2.9(\mathrm{t}),(4 \mathrm{H}) ; 3.5(\mathrm{t}),(4 \mathrm{H}) ; 6.7(\mathrm{~d}),(1 \mathrm{H}),(\mathrm{CH}=\mathrm{CHCO}), \mathrm{J}=15.70 \mathrm{HZ} ; 6.8(\mathrm{~d}),(1 \mathrm{H})$, $\left(\mathrm{CH}=\mathrm{CHC}_{6} \mathrm{H}_{5}\right) \mathrm{J}=15.70 \mathrm{HZ} ; 7.3-7.4(\mathrm{~m}),(4 \mathrm{H})$.

Ii- IR $\left(\mathrm{cm}^{-1}\right) 1654,1546$

1H NMR-( $\delta)-2.2(\mathrm{~s}),(3 \mathrm{H}), \mathrm{NMe} ; 2.9(\mathrm{t}),(4 \mathrm{H}) ; 3.5(\mathrm{t}),(4 \mathrm{H}) ; 6.7(\mathrm{~d}),(1 \mathrm{H}),(\mathrm{CH}=\mathrm{CHCO}), \mathrm{J}=15.70 \mathrm{HZ} ; 6.8(\mathrm{~d}),(1 \mathrm{H})$, $\left(\mathrm{CH}=\mathrm{CHC}_{6} \mathrm{H}_{5}\right) \mathrm{J}=15.70 \mathrm{HZ} ; 7.3-7.4(\mathrm{~m}),(4 \mathrm{H}) ; 2.5(\mathrm{~s}),(6 \mathrm{H}), \mathrm{NMe}_{2}$.

Ij- IR $\left(\mathrm{cm}^{-1}\right) 1656,1680,2550$

1H NMR- $(\delta)-2.2(\mathrm{~s}),(3 \mathrm{H}), \mathrm{NMe} ; 2.9(\mathrm{t}),(4 \mathrm{H}) ; 3.5(\mathrm{t}),(4 \mathrm{H}) ; 6.7(\mathrm{~d}),(1 \mathrm{H}),(\mathrm{CH}=\mathrm{CHCO}), \mathrm{J}=15.70 \mathrm{HZ} ; 6.8(\mathrm{~d}),(1 \mathrm{H})$, $\left(\mathrm{CH}=\mathrm{CHC}_{6} \mathrm{H}_{5}\right) \mathrm{J}=15.70 \mathrm{HZ} ; 7.3-7.4(\mathrm{~m}),(4 \mathrm{H}) ; 5.6(\mathrm{~s}),(1 \mathrm{H}), \mathrm{OH}$.

\section{Conclusion}

The given study clearly showed that all new cinnamamides derivatives were synthesized by using heterocyclic moiety $\mathrm{N}$-methyl piperazine and different aromatic aldehyde gives very good yield. All synthesizes compounds were characterized on the basis of elemental analysis, IR spectra and ${ }^{1} \mathrm{HNMR}$ spectral analysis. Synthesized compounds have been tested for antimicrobial activities and it gives very good results with increasing the concentration of synthesized compounds.

\section{References}

[1]. Pathan R U and Patil S L, Oriental Journal of Chemistry, Vol 24(2), 2008, 709-712.

[2]. Gregory N, Beatch Cindy, J Longley, Michael J, Walker Richard A Wall, Patent No US7, 687, 536 B2, mar 30, $2010,1-36$.

[3]. Murray P R, Baron E J, Tenover F C, Yolken R, Susceptibility tests of fastidious bacteria.Manual of Clinical Microbiology, American Society for Microbiology, Washington DC, $6^{\text {th }}$ edition, 1995, 1342-1349.

[4]. Ira R. Bacteriology, Standard Operative procedure manual for microbiology laboratories, National Institute of Biologicals 1995,73-97.

[5]. Murray P R, Baron E J, Pfaller M A, Tenover F C, Yolken R, American Society for Microbiology, Washington DC, 1999,14691473.

[6]. Yumei Xiao, Yang BoLi, Huizhu Yuan, Shuqing Wan, Yanjun Xu and Zhaohai Qin Molecules, 16, $2011,8945-8957$.

[7]. Kakwani Manoj D Desai Palsule Lele Arundhati C Ray Muktikant Rajan M G, Bioorganic \& Medicinal Chemistry Letters, Vol 21, 2011, 6523-6526.Wu Linbo Jin Chunli and Sun Xiangying, Biomacromolecules, 12 (1), 2011, 235-241.

[8]. Jin Chunli Sun Xiangying Wu Linbo, Designed Monomers \& Polymers, Volume 14, 2011, 47-55.

[9]. Junli C and Cchan T H, Comprehensive Organic Reactions in Aqueous Media, John Wiley \& Sons, Inc 2007, $2^{\text {nd }}$ edition.

[10]. Kong J O, Lee S M and Young-J, J Nematol, 39, (1), 2007, 31-36.De P, Baltas M and Bedos F, Current Med Chemistry, 18, $2011,1672-1703$ 